\title{
Eslicarbazepine Acetate: A New Improvement on a Classic Drug Family for the Treatment of Partial-Onset Seizures
}

\author{
Graciana L. Galiana $^{1}$ - Angela C. Gauthier ${ }^{1}$ (I) Richard H. Mattson $^{1}$
}

Published online: 24 July 2017

(C) The Author(s) 2017. This article is an open access publication

\begin{abstract}
Eslicarbazepine acetate is a new anti-epileptic drug belonging to the dibenzazepine carboxamide family that is currently approved as adjunctive therapy and monotherapy for partial-onset (focal) seizures. The drug enhances slow inactivation of voltage-gated sodium channels and subsequently reduces the activity of rapidly firing neurons. Eslicarbazepine acetate has few, but some, drugdrug interactions. It is a weak enzyme inducer and it inhibits cytochrome P450 2C19, but it affects a smaller assortment of enzymes than carbamazepine. Clinical studies using eslicarbazepine acetate as adjunctive treatment or monotherapy have demonstrated its efficacy in patients with refractory or newly diagnosed focal seizures. The drug is generally well tolerated, and the most common side effects include dizziness, headache, and diplopia. One of the greatest strengths of eslicarbazepine acetate is its ability to be administered only once per day. Eslicarbazepine acetate has many advantages over older antiepileptic drugs, and it should be strongly considered when treating patients with partial-onset epilepsy.
\end{abstract}

Angela C. Gauthier

angela.gauthier@yale.edu

1 Department of Neurology, Yale Comprehensive Epilepsy

Center, Yale School of Medicine, PO Box 208018,

New Haven, CT 06520, USA

\section{Key Points}

Eslicarbazepine acetate is an effective and safe treatment option for partial-onset seizures as adjunctive therapy and monotherapy.

Eslicarbazepine acetate improves upon its predecessors, carbamazepine and oxcarbazepine, by being available in a once-daily regimen, interacting with a smaller range of drugs, and causing less side effects.

\section{Introduction}

Epilepsy is a common neurological disorder affecting over 70 million people worldwide [1]. The condition is defined by having at least two unprovoked seizures $24 \mathrm{~h}$ apart, although it also includes people who have had only one seizure if they have a similar probability of recurrence as an individual with epilepsy [2]. The most frequent type of seizure is the partial-onset (focal) seizure, which is characterized by an initial activation of only part of one cerebral hemisphere [3]. These seizures may or may not be associated with impaired awareness or tonic-clonic convulsions.

Carbamazepine was discovered serendipitously in the 1950s by medicinal chemists at R. G. Geigy, Ltd who were attempting to develop a neuroleptic like the 'blockbuster', chlorpromazine [4]. They replaced the sulfur in chlorpromazine with a $\mathrm{CH}-\mathrm{CH}$. Instead of discovering a new neuroleptic, the chemists had synthesized imipramine, a 
major antidepressant. Further studies to develop a neuroleptic were made on other tricyclic carbomoyl compounds. They subsequently and unintentionally developed carbamazepine, a new and unique anti-epileptic drug (AED) [5].

Studies in Europe in the 1960s established the AED properties of carbamazepine, and it soon became widely used for partial-onset seizures. However, concerns about hematological toxicity led to a delay of almost a decade before carbamazepine was approved for use in USA. Later, Mattson et al. in two large, randomized, double-blind, prospective monotherapy clinical trials found carbamazepine to be as good, and by some measures, better, than phenobarbital, primidone, and valproate. Efficacy was not better than phenytoin, but other studies suggested that phenytoin caused more adverse effects, including neuropsychological problems [6-8]. Soon afterward, carbamazepine became the 'gold standard,' and most clinical trials of new AEDs were compared with it.

Adverse effects and complex pharmacokinetics were limitations in the use of carbamazepine. Oxcarbazepine, the keto analog of carbamazepine introduced in the 1980s by CIBA-Geigy, had improved pharmacokinetics, but it still had a relatively short half-life and limited formulations initially. Eventually, it was discovered that the reduced metabolite and specifically the S-enantiomer, eslicarbazepine, was probably the main active AED and that oxcarbazepine was likely a pro-drug.

Despite the introduction of many new AEDs over the past three decades, seizures remain uncontrolled in approximately one third of patients [9]. It is important to develop new AEDs that are effective, well tolerated, and safe, with minimal adverse effects and drug-drug interactions. Once-daily dosing can optimize patient compliance. Eslicarbazepine acetate (ESL) is a new AED taken once per day that is approved as add-on and monotherapy for adults with partial-onset seizures [3, 10]. Its structure is similar to carbamazepine and oxcarbamazepine, but its differences help improve upon the weaknesses associated with these compounds. Eslicarbazepine acetate has so far shown encouraging results as an efficacious and safe alternative for treating partial-onset seizures.

\section{Chemical Structure}

Like carbamazepine and oxcarbazepine, ESL is a member of the dibenzazepine carboxamide family (Fig. 1). However, its structural distinction lies in the hydroxy group rather than the keto group in the 10 position of the ring [11]. This allows ESL to have a different stereoselective metabolism. Unlike carbamazepine, ESL is not metabolized to carbamazepine-10,11-epoxide, which can cause adverse effects. This metabolic change may be responsible for the safer profile of ESL, minimizing the enzymatic induction of the cytochrome P450 (CYP) system and autoinduction [12].

The metabolic pathway of ESL has probable advantages compared with oxcarbazepine. The breakdown products of oxcarbazepine consist of $80 \%$ (S)-licarbazepine (eslicarbazepine) and $20 \%$ (R)-licarbazepine, in a ratio of $4: 1$ (Fig. 2). However, ESL is directly metabolized to only (S)licarbazepine, which subsequently undergoes a minor conversion to oxcarbazepine (Fig. 3). Part of the oxcarbazepine is then converted to ( $\mathrm{R}$ )-licarbazepine, making the overall (S)-licarbazepine to (R)-licarbazepine ratio 20:1 [13]. Both of these products are active compounds, but (S)licarbazepine is thought to be more effective, less toxic, and more efficient at crossing the blood-brain barrier than (R)-licarbazpine [14].

\section{Mechanism of Action}

Eslicarbazepine acetate works by inhibiting voltage-gated sodium channels (VGSC), especially in rapidly firing neurons [15]. It has a much lower affinity for VGSC in the resting state compared with carbamazepine and oxcarbazepine [16].
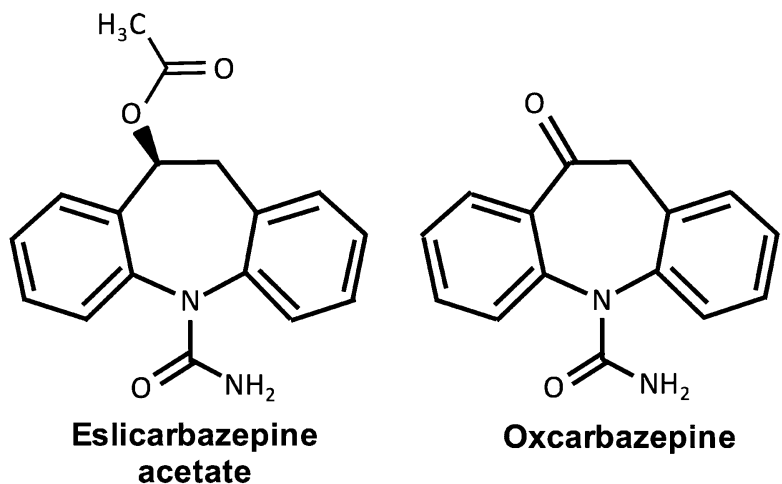

Oxcarbazepine
Fig. 1 Chemical structure of the dibenzazepine-carboxamide family. Eslicarbazepine shares a similar structure with its family members, carbamazepine and oxcarbazepine. However, the difference lies in the 5-carboxamide attached at the 10, 11 position

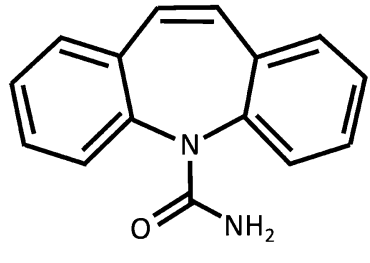

Carbamazepine 


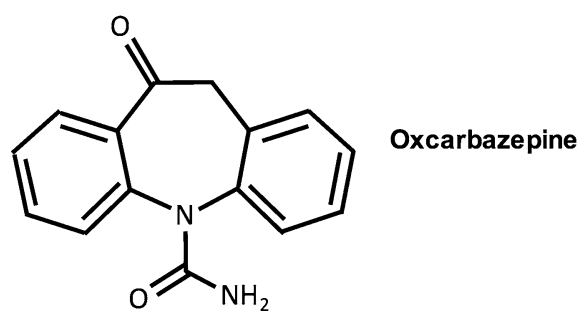

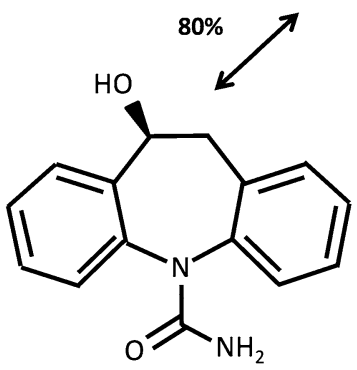

(S)-licarbazepine

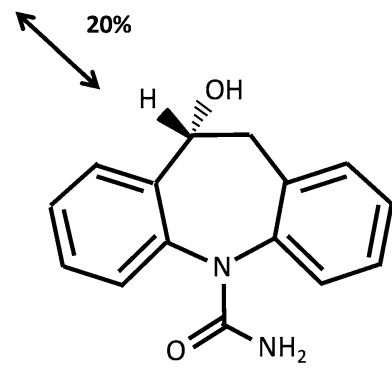

(R)-licarbazepine
Fig. 2 Metabolic pathway of oxcarbazepine. Oxcarbazepine gets metabolized to (S)-licarbazepine and (R)-licarbazepine in a 4:1 ratio

Eslicarbazepine acetate also reduces VGSC availability by increasing slow inactivation of VGSCs [16]. Slow inactivation involves structural rearrangement of the pore, and it is believed to regulate channel excitability [17]. This novel mechanism contrasts with traditional VGSC blockers such as carbamazepine, oxcarbazepine, and phenytoin, which all interfere with fast inactivation pathways [18]. In fast inactivation, the VGSC pore is occluded by a cytoplasmic region [19]. This is thought to play a role in action potential termination and refractory period regulation.

Finally, ESL inhibits $\mathrm{Ca}_{\mathrm{v}} 3.2 \mathrm{~T}$-type $\mathrm{Ca}^{2+}$ channels [15]. Deletion of these channels has been shown to decrease spontaneous seizures and remove the neuropathological markers of chronic epilepsy in mice [20]. Blocking these epileptogenic channels enhances the ability of ESL to further prevent seizures.

\section{Pharmacokinetics}

After ingestion of ESL, the bioavailability of eslicarbazepine is approximately $94 \%$, and $30 \%$ is bound to human plasma proteins $[21,22]$. It has an effective half-life of 20-24 h in the cerebrospinal fluid (Fig. 4) [21, 23]. Peak plasma concentration is attained $2-3 \mathrm{~h}$ after oral administration, and the drug reaches a steady state in the plasma after 4-5 days [21]. Overall, the pharmacokinetics are linear and dose proportional [24]. They remain generally unaffected by age, sex, and food intake [21, 25].

Eslicarbazepine acetate undergoes a quick and nearcomplete first-pass hydrolysis to eslicarbazepine primarily in the liver, although some is metabolized by the intestine
[26]. Because of this, ESL plasma concentrations are too low to be quantified after oral administration. Eslicarbazepine is eliminated through urine mostly in unchanged form $(67 \%)$ or as a conjugate with glucuronic acid (33\%) (Fig. 3) [26]. A very small proportion of eslicarbazepine is converted to oxcarbazepine, which is also excreted in urine either unchanged or glucuronated. This may happen immediately or after breakdown into (S)-licarbazepine and (R)-licarbazepine [21].

Because ESL heavily relies on the kidneys for excretion, moderate-to-severe renal failure can prevent effective clearance of metabolites, necessitating a decreased dosage [27]. However, mild-to-moderate hepatic damage does not seem to significantly affect ESL pharmacokinetics, thus dosage can remain unchanged [26].

There are limited data on the relationship between the pharmacokinetics and pharmacodynamics of ESL. One retrospective observational study investigated pharmacokinetic variability in 168 Norwegian patients taking ESL [28]. These patients were taking a median dose of $800 \mathrm{mg}$ ESL per day (range 400-1600 mg), and they had a median ESL serum concentration of $53 \mu \mathrm{mol} / \mathrm{L}$ (range 13-132 $\mu \mathrm{mol} / \mathrm{L}$ ). They found that there was very broad 25 -fold inter-patient variability in ESL concentration/dose ratios. There was also an extensive range of ESL serum concentrations in patients who attained increased seizure control: 13-99 $\mu \mathrm{mol} / \mathrm{L}$ (median of $57 \mu \mathrm{mol} / \mathrm{L}$ ). This pharmacokinetic variability was not significantly affected by age. The findings suggest that therapy should be individualized, and it is still unclear whether therapeutic drug monitoring is useful in these patients. Another study performed a pharmacokinetic/pharmacodynamic analysis of patients taking ESL [29]. This group concluded that increased eslicarbazepine concentrations correlated with increased seizure reduction with moderate inter-subject variability. This finding was not modulated by concomitant AEDs.

\section{Interactions}

Like oxcarbazepine, ESL is a weak enzyme inducer of CYP3A4 and uridine 5'-diphospho-glucuronosyl, stimulating a smaller range of enzymes than carbamazepine [30]. The effect is not strong enough to warrant dosage changes in AEDs metabolized by these enzymes when co-administered with ESL (Fig. 5) [21]. This gives ESL an advantage over carbamazepine, reducing the number of drug interactions. However, like carbamazepine and oxcarbazepine, ESL may decrease the effectiveness of hormonal contraceptives owing to its induction of CYP3A4, which helps metabolize levonorgestrel and ethinylestradiol (Fig. 6) [31]. Women of childbearing age may need an 
<smiles>CC(=O)O[C@H]1Cc2ccccc2N(C(N)=O)c2ccccc21</smiles>

\section{$61.9 \%$}

Eslicarbazepine acetate

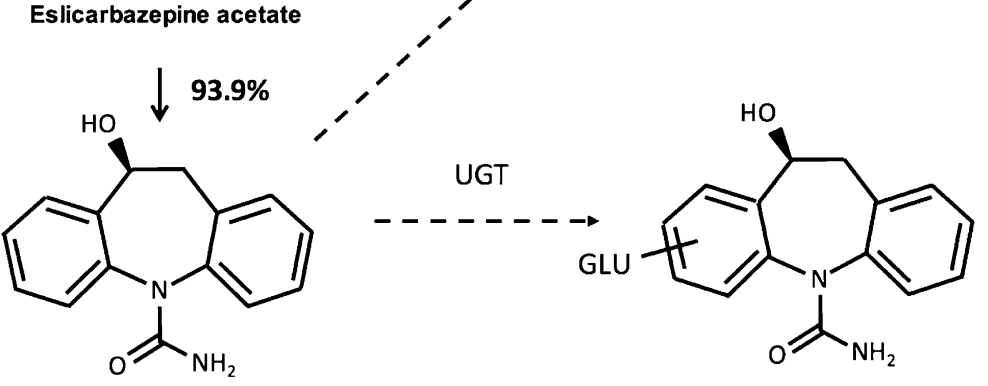

(S)-licarbazepine

(S)-licarbazepine-GLU

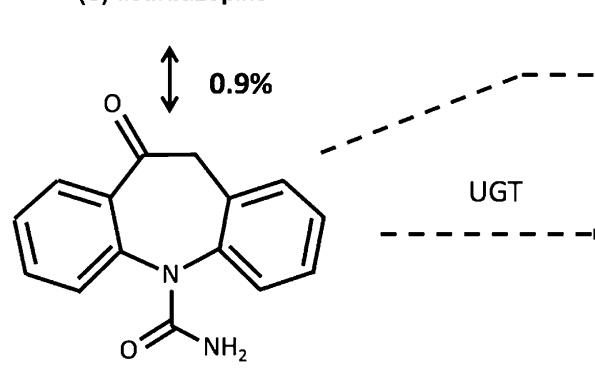

Oxcarbazepine<smiles>NC(=O)N1c2ccccc2C[C@@H](O)c2ccccc21</smiles>

(R)-licarbazepine

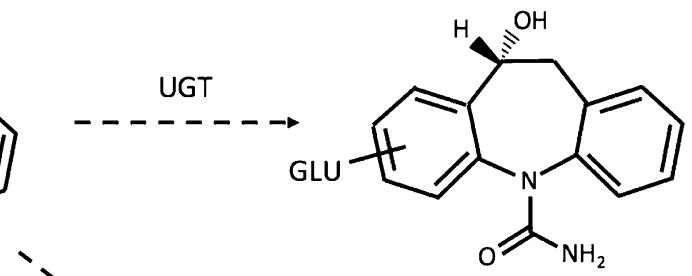

(R)-licarbazepine-GLU

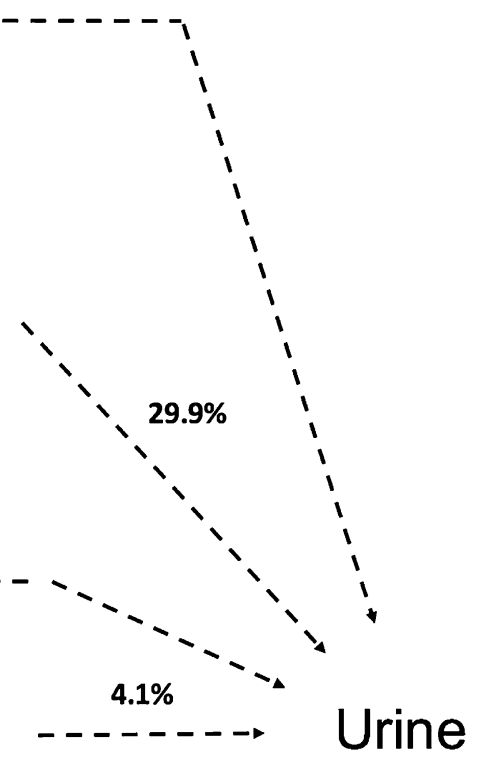<smiles>CC=CC(N)=O</smiles><smiles>CC1CC1(C)S(=O)(=O)I</smiles>

Fig. 3 Metabolic and elimination pathways of eslicarbazepine acetate. Eslicarbazepine acetate gets broken down to mostly (S)licarbazepine. Only a small percentage turns into oxcarbazepine and subsequently (R)-licarbazepine, making the final ratio 20:1. This gives eslicarbazepine acetate an advantage over oxcarbazepine, as

increase in oral contraceptive dose to combat this effect or use alternative contraceptive methods.

Eslicarbazepine acetate also inhibits CYP2C9 and CYP2C19; therefore, drugs metabolized by these enzymes, such as phenytoin, may require a decreased dosage when given concomitantly with ESL [21]. Eslicarbazepine acetate does not appear to interact significantly with
(S)-licarbazepine is more effective, less toxic, and more efficient at crossing the blood-brain barrier than (R)-licarbazepine. Eslicarbazepine and its breakdown products are eventually eliminated in the urine either gluconurated or in unchanged form. GLU glucuronide, UGT uridine glucuronosyltransferase

levitiracetam, gabapentin, valproate, and clobazam [32]. Enzyme inducers such as carbamazapine, phenobarbital, and phenytoin all increase eslicarbazepine clearance by affecting the glucuronidation pathway, resulting in an average decrease of 21-33\% plasma ESL (Fig. 7) [21]. More ESL may be needed when co-administered with these AEDs. Notably, ESL should not be given alongside 


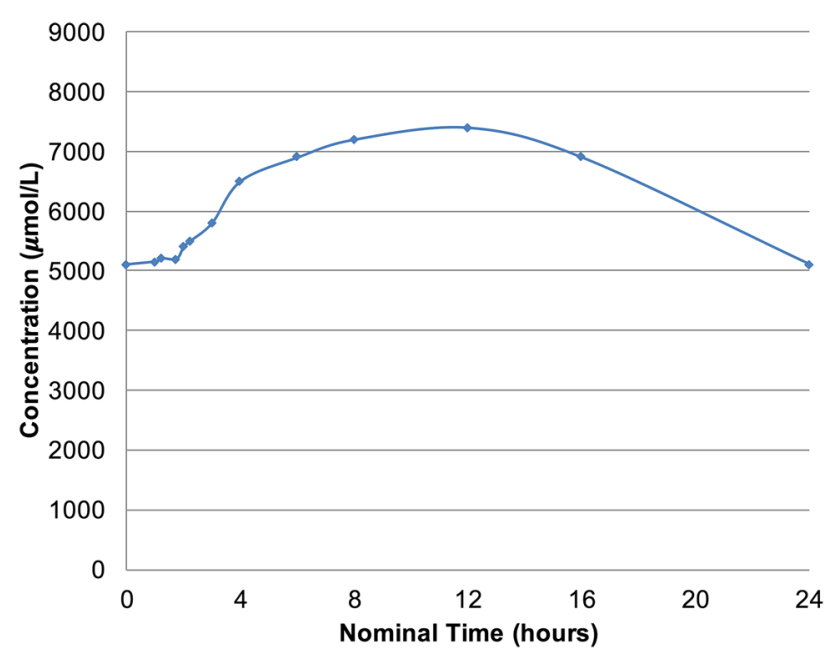

Fig. 4 Concentration of eslicarbazepine acetate in cerebrospinal fluid over time. Mean eslicarbazepine acetate concentration in the cerebrospinal fluid after last-dose administration to six healthy volunteers at $1200 \mathrm{mg} /$ day. According to eslicarbazepine acetate levels reached in the cerebrospinal fluid, this drug is suitable for a once-daily regimen. Data obtained from Nunes et al. [23]

oxcarbazepine because this may cause overexposure to (S)licarbazepine [33].

Eslicarbazepine acetate reduces the levels of mean systemic exposure by $54 \%$ for simvastatin and $36-39 \%$ for rosuvastatin, thus it is advisable to monitor response to therapy (e.g., cholesterol levels) and increase the dose of these drugs if necessary (Fig. 6) [32, 34]. Eslicarbazepine acetate has also been shown to decrease (S)-warfarin levels by $23 \%$, but it has no influence over (R)-warfarin pharmacokinetics or coagulation [35]. It may be prudent to carefully observe the international normalized ratio for patients who have recently started taking ESL while also receiving warfarin. Eslicarbazepine acetate does not seem to have any clinically relevant effect on digoxin or metformin pharmacokinetics [36, 37].

\section{Clinical Studies}

Eslicarbazepine acetate was developed to treat adult partial-onset epilepsy as an adjunctive drug or monotherapy. It is now being investigated in children. Studies consistently show that eslicarbazepine is generally effective at controlling partial-onset seizures, and it remains efficacious in long-term trials.

\subsection{Adjunctive Treatment in Refractory Epilepsy}

A phase II placebo-controlled trial of 143 treatment-refractory patients with partial-onset epilepsy studied the efficacy of ESL at doses gradually titrated to $1200 \mathrm{mg}$ [38]. After 12 weeks of treatment, they found that 54\% of patients treated daily and $41 \%$ of patients treated twice per day responded to therapy (defined as a $\geq 50 \%$ seizure reduction), vs. $28 \%$ in the placebo group. In addition, $24 \%$ patients in each of the treatment groups were seizure free, which was significantly higher than the $9 \%$ of patients in the placebo group.

Three randomized, placebo-controlled, double-blind phase III trials have also been employed to examine ESL $[14,39,40]$. These trials studied a total of 1053 refractory patients from 125 different centers at baseline for 8 weeks, then randomized them to receive a placebo or once daily ESL at 400,800 , or $1200 \mathrm{mg}$. Eslicarbazepine acetate treatment began at $400 \mathrm{mg}$ and either remained there or was titrated to the higher doses over 2 weeks. Seizure frequency and adverse effects were then monitored over a 12-week maintenance period. Responder rates varied from
Fig. 5 Effect of eslicarbazepine acetate on other anti-epileptic drugs. Co-administration of eslicarbazepine acetate with phenytoin may require a decreased dosage of phenytoin. However, when eslicarbazepine acetate is given with gabapentin, lamotrigine, levitiracetam, phenobarbital, topiramate, carbamazepine, or valproate, the dosages of these drugs can remain unchanged. Data obtained from Aptiom prescribing information [33]. CI confidence interval

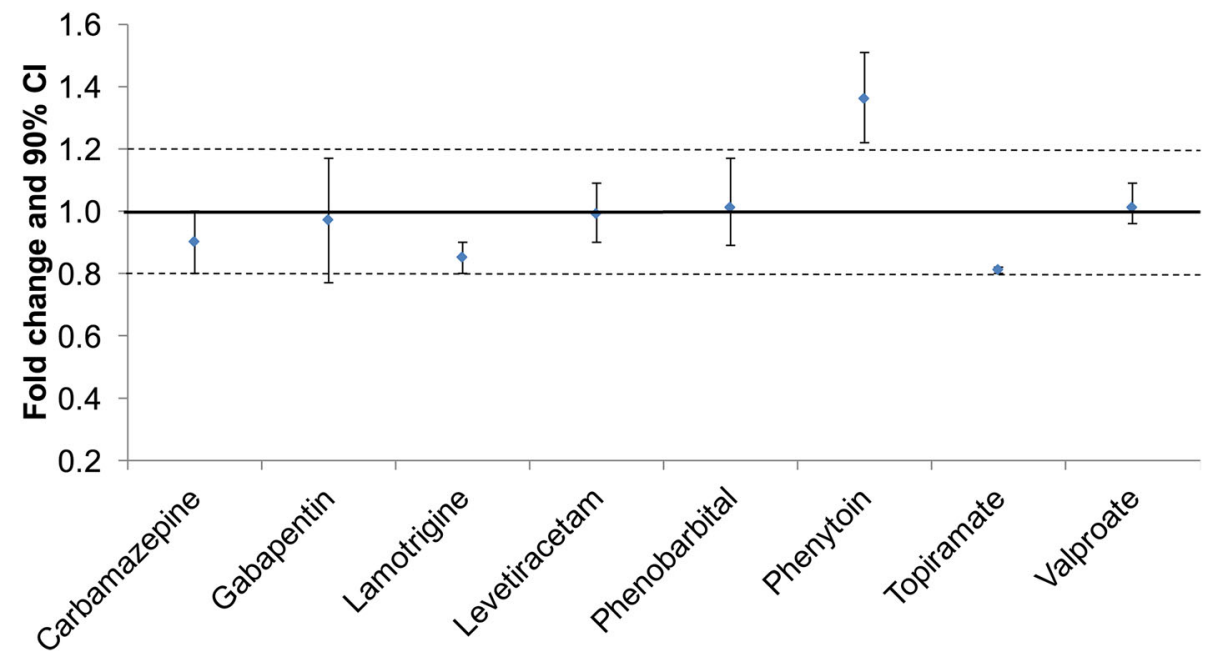




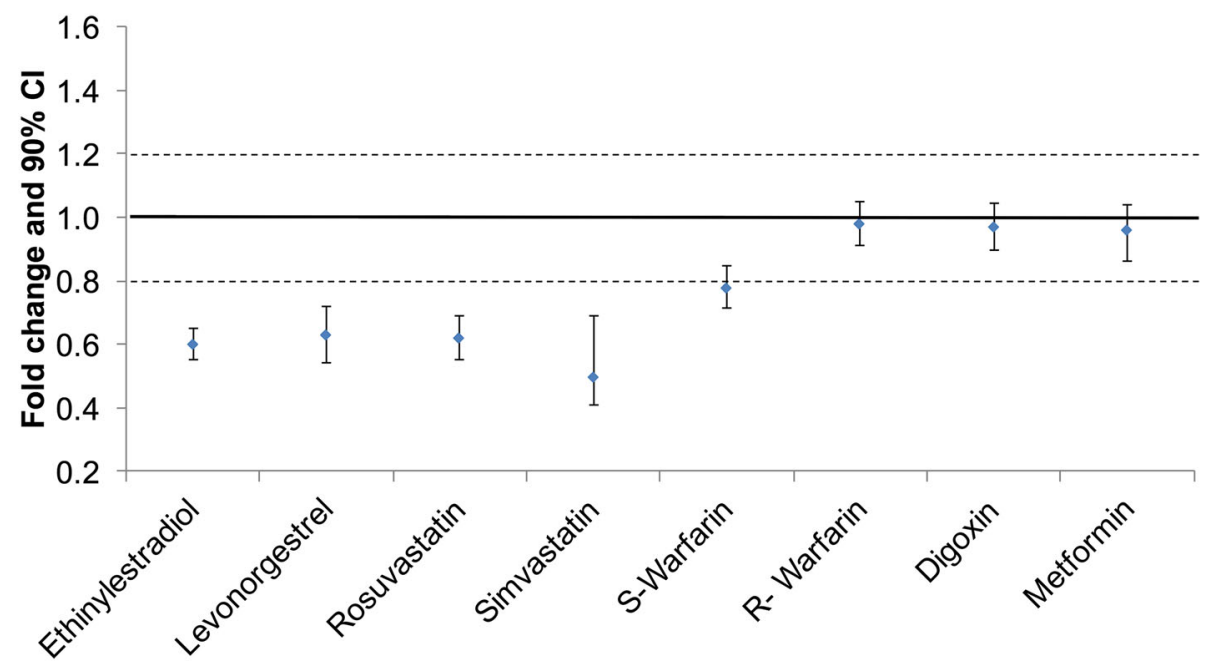

Fig. 6 Effect of eslicarbazepine acetate on other drugs. Eslicarbazepine acetate increases the clearance of oral contraceptives, requiring either an increase in oral contraceptive dose or a change to a non-hormonal birth control method. It also increases clearance of simvastatin and rosuvastatin, thus a dosage increase in these drugs may be necessary if there is a significant change in lipid levels. No dosage adjustments are needed when eslicarbazepine acetate is given with digoxin or metformin. Data obtained from Aptiom prescribing information [33]. $C I$ confidence interval

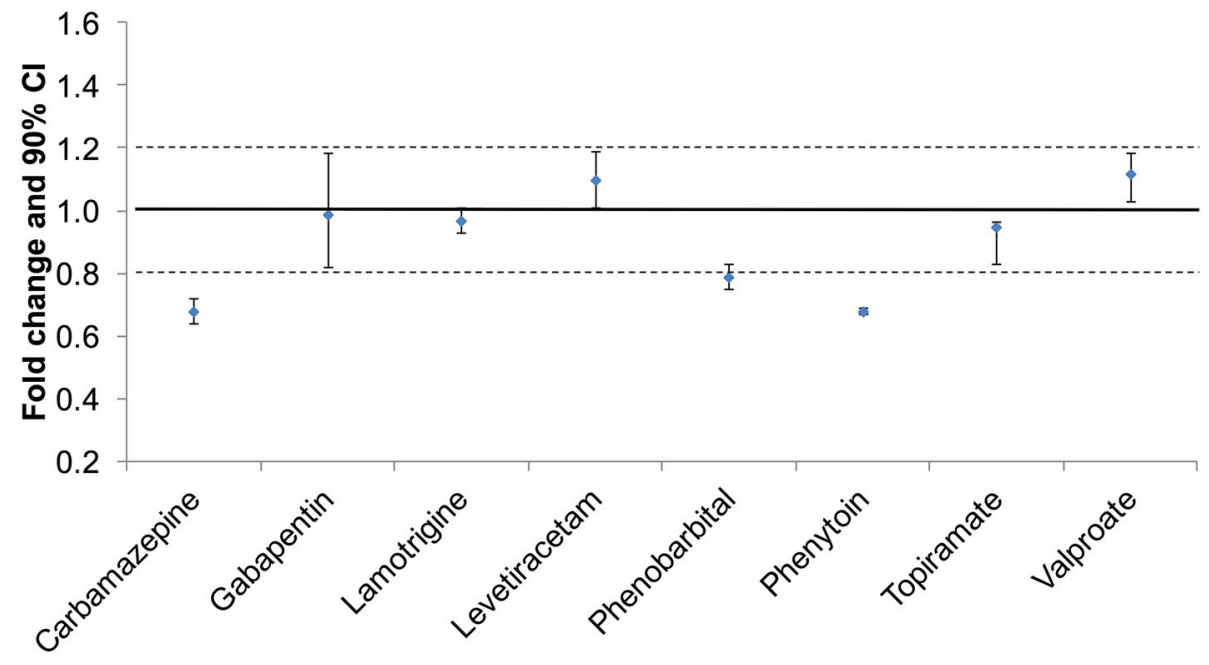

Fig. 7 Effect of other anti-epileptic drugs on eslicarbazepine acetate. When eslicarbazepine acetate is co-administered with carbamazepine, phenobarbital, or phenytoin, the dose of eslicarbazepine acetate may need to be increased because these drugs increase clearance of eslicarbazepine acetate. However, co-administration of

16.7 to $23 \%$ for $400 \mathrm{mg} /$ day, $35-40 \%$ for $800 \mathrm{mg} /$ day, $37.1-45 \%$ for $1200 \mathrm{mg} /$ day, and $13-23 \%$ for placebo $[14,39,40]$. All three studies found that seizure frequency was significantly lower in the 800- and 1200-mg groups, but not the 400-mg group. Adverse effects were all mild or moderate in severity, the most common ones being dizziness, headache, and diplopia. A pooled analysis of these patients found that the median decrease in seizure frequency was $35 \%$ at $800 \mathrm{mg} / \mathrm{day}, 39 \%$ at $1200 \mathrm{mg} / \mathrm{day}$, and $15 \%$ with the placebo [41]. This statistically significant eslicarbazepine acetate with gabapentin, lamotrigine, levitiracetam, topiramate, or valproate does not require any ESL dosage adjustments. Data obtained from Aptiom prescribing information [33]. CI confidence interval

difference between treatment and placebo was maintained regardless of sex, geographic region, duration of epilepsy, age at diagnosis, type of seizure, and concomitant AED usage.

Another phase III trial recruited 653 refractory patients from 19 countries all around the world [42]. Patients were given a placebo or ESL at 800 or $1200 \mathrm{mg} /$ day for 12 weeks. They found significant differences in seizure frequency and response rates between the 1200-mg/day and placebo groups but not between the 800-mg/day and 
placebo groups [42]. However, this may be attributed to a relatively higher responder rate in the placebo group compared with other studies. A seizure severity questionnaire was administered to 547 of these patients on day 1 of treatment, 8 weeks into treatment, and at the end of the study [43]. The total score was found to be significantly decreased in both treatment groups compared with the placebo, showing that ESL indeed produced clinically relevant improvements in seizure severity.

\subsection{Long-Term Studies}

Eslicarbazepine acetate maintains its effectiveness over time, as demonstrated by extension and retrospective studies. Two open-label extension studies on patients with partial-onset epilepsy followed patients a year out from two of the phase III trials described above [14, 39, 44, 45]. A total of 462 patients participated in the studies, and responder rates measured every 12 weeks varied between 38 and $53 \%$ after the first 4 weeks of treatment $[44,45]$. The proportion of patients experiencing no seizures in each 12-week block ranged between 5 and $12.5 \%$. Adverse events affected 51-83\% of patients, but most of these were only mild to moderate. Overall, quality-of-life and depression scores were significantly better at the end of the year compared with baseline.

A retrospective study of 327 patients taking ESL in a clinical setting over 1 year found that the responder rate was $46.3 \%$ at 3 months, $57.9 \%$ at 6 months, and $52.5 \%$ at 12 months, while the percentage of patients who were seizure free was 21,28 , and $25 \%$, respectively [46]. In addition, a multicenter retrospective study conducted in Spain followed 253 patients with partial-onset epilepsy for 1 year [47]. These patients had all failed their first trial of AED monotherapy and were subsequently started on ESL. At the end of the year, $62.3 \%$ of patients had experienced no seizures for the last 6 months, and $37.3 \%$ had not had any seizures for the last year. In fact, after initiating treatment with ESL, $54.2 \%$ of patients completely withdrew their prior AED and switched to ESL monotherapy. Finally, another retrospective study investigated 152 patients over 2 years [48]. Responder rates were decreased compared with other studies; they were 25.7, 25.7, 19.0 , and $17.1 \%$ at $6,12,18$, and 24 months, respectively. However, the authors still concluded that ESL was clinically helpful and well tolerated as adjunctive therapy in patients with refractory partial-onset seizures.

\subsection{Monotherapy}

Eslicarbazepine acetate monotherapy trials have generally found that the drug performs well. Two randomized, historical-control, phase III studies followed a total of 365 refractory patients for an 8-week baseline and then converted them to receive either 1200 or $1600 \mathrm{mg}$ of ESL per day as

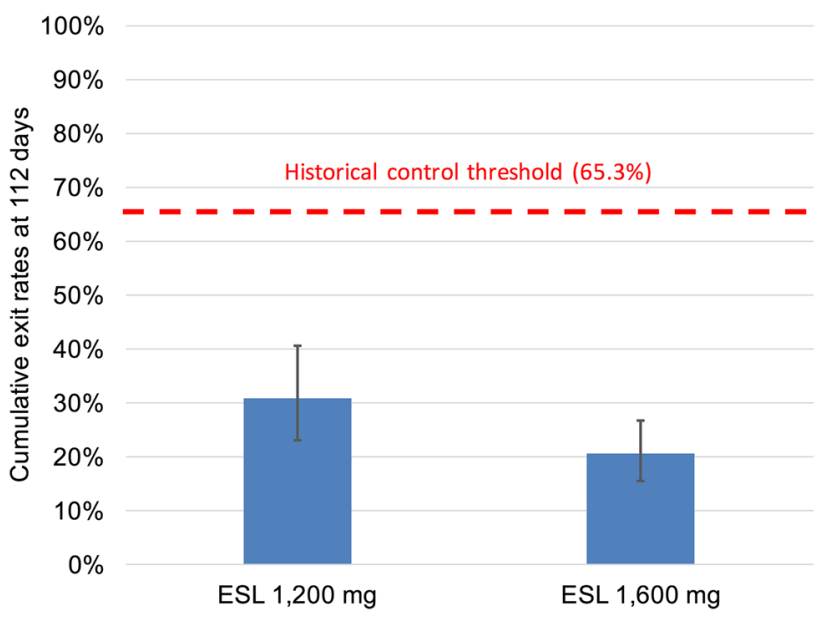

Fig. 8 Kaplan-Meier analysis of estimated exit rates of the integrated population. This pooled analysis of two phase III studies shows that the upper $95 \%$ confidence limits (upper error bars) for the exit rates of patients receiving eslicarbazepine acetate (ESL) monotherapy at 1200 and $1600 \mathrm{mg}$ were both below the historical threshold of $65.3 \%$ [51]. Patients exited the study when they met established exit criteria, which indicates deteriorating seizure control

monotherapy for 18 weeks $[49,50]$. They measured exit rates in each group, defined by declining seizure control. A pooled analysis of the studies revealed that the exit rate was $30.8 \%$ for the ESL $1200-\mathrm{mg} /$ day group, $20.6 \%$ for the ESL $1600-\mathrm{mg}$ /day group, and $65.3 \%$ for the historical control (Fig. 8) [51]. The difference between treatment groups and the historical control was statistically significant, indicating that ESL monotherapy was effective and safe.

Eslicarbazepine acetate monotherapy has also been revealed to be non-inferior to controlled-release carbamazepine in a phase III trial [52]. This study followed 815 newly diagnosed patients randomized to receive once-daily ESL or twice-daily controlled-release carbamazepine for 26 weeks. Patients were started at a dose of $800 \mathrm{mg} /$ day of ESL or $200 \mathrm{mg}$ of carbamazepine twice daily. Dosage was increased to $1200 \mathrm{mg} / \mathrm{day}$ of ESL and $400 \mathrm{mg}$ of carbamazepine twice daily and possibly to $1600 \mathrm{mg}$ /day of ESL and $600 \mathrm{mg}$ of carbamazepine twice daily if seizures occurred. The proportion of patients who were seizure free for at least 6 months was $71.1 \%$ in the ESL group and $75.6 \%$ in the carbamazepine group. In addition, $64.7 \%$ of patients on ESL did not experience seizures for the whole year, compared with $70.3 \%$ on carbamazepine. The study concluded ESL once daily was not inferior to twice-daily carbamazepine in patients recently diagnosed with epilepsy.

\subsection{Data in Children}

There is presently only one clinical study on eslicarbazepine in children, but it shows promising results. In this 
open-label study, 29 pediatric patients aged 2-17 years with treatment-refractory epilepsy were given ESL at $5 \mathrm{mg} /$ $\mathrm{kg} /$ day on weeks $1-4,15 \mathrm{mg} / \mathrm{kg} /$ day on weeks $5-8$, and 30 $\mathrm{mg} / \mathrm{kg} / \mathrm{day}$ on weeks 9-12 [53]. They found that the children experienced similar rates of metabolism to adults, but the younger children cleared the drug more quickly than the adolescents. Pediatric patients aged 2-7 years and 12-17 years experienced a dose-dependent reduction in the frequency of seizures, but this did not occur in the 7- to 11-year-old age group [53]. Overall, the drug was well tolerated, and most adverse effects were of mild intensity. The patients experienced a total of 34 drug-related adverse events, with increased frequency at the higher doses. The only severe effects were two cases of seizure worsening that occurred at a dose of $30 \mathrm{mg} / \mathrm{kg} / \mathrm{day}$, which led to drug discontinuation [53].

An ongoing phase III clinical trial is currently evaluating ESL for refractory partial seizures in children (Clinical Trials.gov Identifier: NCT00988156) [32]. Further data are needed to definitively determine whether eslicarbazepine is effective and safe in the pediatric population, but early results are encouraging.

\section{Safety}

Eslicarbazepine is generally considered a safe and welltolerated drug. The most frequent adverse effects include dizziness, headache, fatigue, and diplopia [14, 39, 40, 49]. Somnolence, abnormal coordination, vomiting, and blurred vision may also occur, but most of the events are only of mild or moderate severity [54]. Data collected on 247 patients who were taking eslicarbazepine as an add-on therapy found that $3.7 \%$ of patients experienced a serious adverse event, such as hyponatremia and allergic skin reactions [55].

Long-term studies have demonstrated consistent findings. In a 1-year open-label extension study, $51 \%$ of 314 patients experienced adverse effects as result of eslicarbazepine, and $97 \%$ of them were classified as mild to moderate severity [44]. Adverse events were most common during the first 3 months of treatment (35\% incidence) compared with a $12.7 \%$ incidence during the last 3 months of the study [44]. Only $3.5 \%$ of patients stopped therapy because of these events. Analysis of $434,468.3$ patientmonths between 1 October, 2009 and 21 October, 2013 in 18 different European countries found spontaneous reports of 367 serious and 509 non-serious adverse effects [56]. Among these, hyponatremia was most common (114 reports), followed by convulsion (48 reports) and dizziness (29 reports).

Hyponatremia is an important side effect of some AEDs, especially oxcarbazepine and carbamazepine [57, 58].
Eslicarbazepine seems to have a lower incidence of hyponatremia than these drugs, occurring in only $0.6-1.5 \%$ of patients $[42,59,60]$. A randomized, double-blind, placebo-controlled phase III trial of 653 patients found that $1.5 \%$ of patients taking eslicarbazepine developed hyponatremia, compared with $0 \%$ on placebo [42]. A reduction in sodium level mostly occurred within 8 weeks of treatment and then leveled out. The hyponatremia was severe enough to cause discontinuation in $1.4 \%$ of patients taking $1200 \mathrm{mg}$ of eslicarbazepine, but none of the patients taking $800 \mathrm{mg}$ dropped out for this reason [42]. Notably, ESL causes higher rates of hyponatremia in elderly poststroke seizure patients. A 2-year observational study of 32 such patients taking between $400 \mathrm{mg}$ and $1200 \mathrm{mg}$ of ESL per day found that hyponatremia developed in $12.5 \%$, warranting serum sodium level monitoring in this population [61].

Eslicarbazepine may cause dermatological side effects, but the incidence is rare. According to three randomized placebo-controlled studies, the most common skin reactions were rash, alopecia, and hyperhidrosis, which occurred in $0.5-3.2 \%$ of patients $[14,39,42]$. Serious dermatological reactions included three cases of drug reaction with eosinophilia and systemic symptoms and one case of Stevens-Johnson syndrome [14, 39, 42].

Eslicarbazepine has proven superior or at least comparable to oxcarbazepine and carbamazepine in other safety profiles as well. Ley et al. studied 108 patients on eslicarbazepine, of which $52 \%$ were previously taking carbamazepine or oxcarbazepine [54]. They found that the average low-density lipoprotein, total cholesterol, and triglyceride values of the group significantly decreased after starting eslicarbazepine [54]. In many cases, they went from pathological to benign readings. Liver function tests remained similar before and after starting eslicarbazepine. Additionally, unlike carbamazepine, ESL does not lower the leukocyte count. Although it is a relatively new drug, eslicarbazepine has so far demonstrated promising results in safety, with mostly a few mild adverse effects soon after starting treatment.

\section{Dosage Regimens and Combinations}

Most patients should start at a dosage of $400 \mathrm{mg}$ of ESL per day [33]. However, dosage may begin at $800 \mathrm{mg} /$ day if seizures are so severe or frequent that reducing them outweighs the cost of adverse events. After initiating therapy, the daily dose may be raised by $400-600 \mathrm{mg}$ per week, depending on how the patient responds to treatment [33]. Target maintenance dose is $800 \mathrm{mg} / \mathrm{day}$, but this may be increased to a maximum of $1600 \mathrm{mg} /$ day if needed. Patients with moderate-to-severe renal deficiency should 
start treatment at $200 \mathrm{mg} /$ day with a maintenance dose maximum of $600 \mathrm{mg} / \mathrm{day}$. Patients with mild-to-moderate hepatic damage do not need dosage modification, but those with severe damage possibly should not take ESL because the drug has not been studied in this population [33].

If other AEDs are being used concomitantly with ESL, some evidence suggests that those AEDs should have a different mechanism of action to increase efficacy and reduce side effects. Levitiracetam is a good option for a combination. However, taking ESL with phenobarbital, valproate, or drugs with a similar effect on VGSCs such as lacosamide or lamotrigine increases the frequency of instability, dizziness, and nausea [62]. If patients taking oxcarbazepine or carbamazepine elect to switch to ESL, an oxcarbazepine:ESL dose ratio of 1:1 may be used to transition medications overnight [63]. However, if the patient is switching from carbamazepine to ESL, a carbamazepine:ESL dose ratio of $1: 1.3$ is suggested [63]. The conversion period should last at least 1-2 weeks, and it may be necessary to also modify concomitant medications metabolized by CYP enzymes.

\section{Conclusions}

Eslicarbazepine acetate is a recently approved AED that is highly effective and safe in the treatment of adult partialonset seizures. The drug possesses several advantages over other members of the dibenzapine-carboxamide family, carbamazepine and oxcarbamazepine. Its pharmacological profile allows for once-daily dosage, which may help promote patient adherence. In addition, ESL is a weaker enzyme inducer than carbamazepine, reducing the number of drug interactions. Eslicarbazepine acetate is also less likely to cause hyponatremia than carbamazepine and oxcarbamazepine. It is not inferior to carbamazepine in efficacy, and adverse effects are predominantly mild to moderate. Data in children have also shown promising results. Eslicarbazepine acetate is a solid option in the treatment of partial-onset epilepsy as adjunctive therapy and monotherapy, and it has the potential to greatly improve quality of life for treatment-refractory patients.

\section{Compliance with Ethical Standards}

Funding No sources of funding were received for the preparation of this article.

Conflict of interest Graciana L. Galiana, Angela C. Gauthier, and Richard H. Mattson have no conflicts of interest directly relevant to the content of this article.

Open Access This article is distributed under the terms of the Creative Commons Attribution-NonCommercial 4.0 International License (http://creativecommons.org/licenses/by-nc/4.0/), which permits any noncommercial use, distribution, and reproduction in any medium, provided you give appropriate credit to the original author(s) and the source, provide a link to the Creative Commons license, and indicate if changes were made.

\section{References}

1. Singh A, Trevick S. The epidemiology of global epilepsy. Neurol Clin. 2016;34(4):837-47.

2. Fisher RS, Acevedo C, Arzimanoglou A, Bogacz A, Cross JH, Elger CE, et al. ILAE official report: a practical clinical definition of epilepsy. Epilepsia. 2014;55(4):475-82.

3. Zelano J, Ben-Menachem E. Eslicarbazepine acetate for the treatment of partial epilepsy. Expert Opin Pharmacother. 2016;17(8):1165-9.

4. Mattson RH. Carbamazepine. In: Engel J, Pedley T, editors. Epilepsy: a comprehensive textbook. Philadelphia: LippincottRaven Publishers; 1997.

5. Galbraith A. Tegretol-background and introduction. In: Wink C, editor. Tegretol in epilepsy. Manchester: C. Nicholls \& Co.; 1972. p. 132-3.

6. Mattson RH, Cramer JA, Collins JF, Smith DB, Delgado-Escueta AV, Browne TR, et al. Comparison of carbamazepine, phenobarbital, phenytoin, and primidone in partial and secondarily generalized tonic-clonic seizures. $\mathrm{N}$ Engl $\mathrm{J}$ Med. 1985;313(3):145-51.

7. Mattson RH, Cramer JA, Collins JF. A comparison of valproate with carbamazepine for the treatment of complex partial seizures and secondarily generalized tonic-clonic seizures in adults: the Department of Veterans Affairs Epilepsy Cooperative Study No. 264 Group. N Engl J Med. 1992;327(11):765-71.

8. Trimble MR. Neurobehavioral effects of anticonvulsants. JAMA. 1991;265(10):1307-8.

9. Kwan P, Brodie MJ. Early identification of refractory epilepsy. N Engl J Med. 2000;342(5):314-9.

10. Mattson R, Gauthier A. A treatment option for partial-onset seizures. Neurol Rev. 2017.

11. Banach M, Borowicz KK, Czuczwar SJ. Pharmacokinetic/pharmacodynamic evaluation of eslicarbazepine for the treatment of epilepsy. Expert Opin Drug Metab Toxicol. 2015;11(4):639-48.

12. Hainzl D, Parada A, Soares-da-Silva P. Metabolism of two new antiepileptic drugs and their principal metabolites $\mathrm{S}(+)$ - and $\mathrm{R}(-$ )-10,11-dihydro-10-hydroxy carbamazepine. Epilepsy Res. 2001;44(2-3):197-206.

13. Elger C, Bialer M, Falcao A, Vaz-da-Silva M, Nunes T, Almeida $\mathrm{L}$, et al. Pharmacokinetics and tolerability of eslicarbazepine acetate and oxcarbazepine at steady state in healthy volunteers. Epilepsia. 2013;54(8):1453-61.

14. Ben-Menachem E, Gabbai AA, Hufnagel A, Maia J, Almeida L, Soares-da-Silva P. Eslicarbazepine acetate as adjunctive therapy in adult patients with partial epilepsy. Epilepsy Res. 2010;89(2-3):278-85.

15. Soares-da-Silva P, Pires N, Bonifacio MJ, Loureiro AI, Palma N, Wright LC. Eslicarbazepine acetate for the treatment of focal epilepsy: an update on its proposed mechanisms of action. Pharmacol Res Perspect. 2015;3(2):e00124.

16. Hebeisen S, Pires N, Loureiro AI, Bonifacio MJ, Palma N, Whyment A, et al. Eslicarbazepine and the enhancement of slow inactivation of voltage-gated sodium channels: a comparison with carbamazepine, oxcarbazepine and lacosamide. Neuropharmacology. 2015;89:122-35.

17. Vilin YY, Ruben PC. Slow inactivation in voltage-gated sodium channels: molecular substrates and contributions to channelopathies. Cell Biochem Biophys. 2001;35(2):171-90. 
18. Rogawski MA, Loscher W. The neurobiology of antiepileptic drugs. Nat Rev Neurosci. 2004;5(7):553-64.

19. Goldin AL. Mechanisms of sodium channel inactivation. Curr Opin Neurobiol. 2003;13(3):284-90.

20. Becker AJ, Pitsch J, Sochivko D, Opitz T, Staniek M, Chen CC, et al. Transcriptional upregulation of Cav3.2 mediates epileptogenesis in the pilocarpine model of epilepsy. J Neurosci. 2008;28(49):13341-53.

21. Bialer M, Soares-da-Silva P. Pharmacokinetics and drug interactions of eslicarbazepine acetate. Epilepsia. 2012;53(6):935-46.

22. Fortuna A, Alves G, Falcao A, Soares-da-Silva P. Binding of licarbazepine enantiomers to mouse and human plasma proteins. Biopharm Drug Dispos. 2010;31(5-6):362-6.

23. Nunes T, Rocha JF, Falcao A, Almeida L, Soares-da-Silva P. Steady-state plasma and cerebrospinal fluid pharmacokinetics and tolerability of eslicarbazepine acetate and oxcarbazepine in healthy volunteers. Epilepsia. 2013;54(1):108-16.

24. Perucca E, Elger C, Halasz P, Falcao A, Almeida L, Soares-daSilva P. Pharmacokinetics of eslicarbazepine acetate at steadystate in adults with partial-onset seizures. Epilepsy Res. 2011;96(1-2):132-9.

25. Almeida L, Falcao A, Maia J, Mazur D, Gellert M, Soares-daSilva P. Single-dose and steady-state pharmacokinetics of eslicarbazepine acetate (BIA 2-093) in healthy elderly and young subjects. J Clin Pharmacol. 2005;45(9):1062-6.

26. Almeida L, Potgieter JH, Maia J, Potgieter MA, Mota F, Soaresda-Silva P. Pharmacokinetics of eslicarbazepine acetate in patients with moderate hepatic impairment. Eur J Clin Pharmacol. 2008;64(3):267-73.

27. Maia J, Almeida L, Falcao A, Soares E, Mota F, Potgieter MA, et al. Effect of renal impairment on the pharmacokinetics of eslicarbazepine acetate. Int $\mathbf{J}$ Clin Pharmacol Ther. 2008;46(3):119-30.

28. Svendsen T, Brodtkorb E, Reimers A, Molden E, Saetre E, Johannessen SI, et al. Pharmacokinetic variability, efficacy and tolerability of eslicarbazepine acetate: a national approach to the evaluation of therapeutic drug monitoring data and clinical outcome. Epilepsy Res. 2017;129:125-31.

29. Falcao A, Fuseau E, Nunes T, Almeida L, Soares-da-Silva P. Pharmacokinetics, drug interactions and exposure-response relationship of eslicarbazepine acetate in adult patients with partialonset seizures: population pharmacokinetic and pharmacokinetic/ pharmacodynamic analyses. CNS Drugs. 2012;26(1):79-91.

30. Zaccara G, Perucca E. Interactions between antiepileptic drugs, and between antiepileptic drugs and other drugs. Epileptic Disord. 2014;16(4):409-31.

31. Falcao A, Vaz-da-Silva M, Gama H, Nunes T, Almeida L, Soares-da-Silva P. Effect of eslicarbazepine acetate on the pharmacokinetics of a combined ethinylestradiol/levonorgestrel oral contraceptive in healthy women. Epilepsy Res. 2013;105(3):368-76

32. Zaccara G, Giovannelli F, Cincotta M, Carelli A, Verrotti A. Clinical utility of eslicarbazepine: current evidence. Drug Des Devel Ther. 2015;9:781-9.

33. Pharma International Inc. Aptiom: prescribing information. 2016. http://www.aptiom.com/Aptiom-Prescribing-Information.pdf. Accessed 17 Jul 2017.

34. Falcao A, Pinto R, Nunes T, Soares-da-Silva P. Effect of repeated administration of eslicarbazepine acetate on the pharmacokinetics of simvastatin in healthy subjects. Epilepsy Res. 2013;106(1-2):244-9.

35. Vaz-da-Silva M, Almeida L, Falcao A, Soares E, Maia J, Nunes $\mathrm{T}$, et al. Effect of eslicarbazepine acetate on the steady-state pharmacokinetics and pharmacodynamics of warfarin in healthy subjects during a three-stage, open-label, multiple-dose, singleperiod study. Clin Ther. 2010;32(1):179-92.
36. Vaz da Silva M, Costa R, Soares E, Maia J, Falcao A, Almeida L, et al. Effect of eslicarbazepine acetate on the pharmacokinetics of digoxin in healthy subjects. Fundam Clin Pharmacol. 2009;23(4):509-14.

37. Rocha JF, Vaz-da-Silva M, Almeida L, Falcao A, Nunes T, Santos AT, et al. Effect of eslicarbazepine acetate on the pharmacokinetics of metformin in healthy subjects. Int J Clin Pharmacol Ther. 2009;47(4):255-61.

38. Elger C, Bialer M, Cramer JA, Maia J, Almeida L, Soares-daSilva P. Eslicarbazepine acetate: a double-blind, add-on, placebocontrolled exploratory trial in adult patients with partial-onset seizures. Epilepsia. 2007;48(3):497-504.

39. Elger C, Halasz P, Maia J, Almeida L, Soares-da-Silva P. Efficacy and safety of eslicarbazepine acetate as adjunctive treatment in adults with refractory partial-onset seizures: a randomized, double-blind, placebo-controlled, parallel-group phase III study. Epilepsia. 2009;50(3):454-63.

40. Gil-Nagel A, Lopes-Lima J, Almeida L, Maia J, Soares-da-Silva P. Efficacy and safety of 800 and $1200 \mathrm{mg}$ eslicarbazepine acetate as adjunctive treatment in adults with refractory partial-onset seizures. Acta Neurol Scand. 2009;120(5):281-7.

41. Gil-Nagel A, Elger C, Ben-Menachem E, Halasz P, Lopes-Lima J, Gabbai AA, et al. Efficacy and safety of eslicarbazepine acetate as add-on treatment in patients with focal-onset seizures: integrated analysis of pooled data from double-blind phase III clinical studies. Epilepsia. 2013;54(1):98-107.

42. Sperling MR, Abou-Khalil B, Harvey J, Rogin JB, Biraben A, Galimberti CA, et al. Eslicarbazepine acetate as adjunctive therapy in patients with uncontrolled partial-onset seizures: results of a phase III, double-blind, randomized, placebo-controlled trial. Epilepsia. 2015;56(2):244-53.

43. Cramer JA, Velez FF, Anastassopoulos KP, Bond TC, Gilliam FG, Ryvlin P, et al. Severity and burden of partial-onset seizures in a phase III trial of eslicarbazepine acetate. Epilepsy Behav. 2015;53:149-53.

44. Halasz P, Cramer JA, Hodoba D, Czlonkowska A, Guekht A, Maia J, et al. Long-term efficacy and safety of eslicarbazepine acetate: results of a 1-year open-label extension study in partialonset seizures in adults with epilepsy. Epilepsia. 2010;51(10):1963-9.

45. Hufnagel A, Ben-Menachem E, Gabbai AA, Falcao A, Almeida L, Soares-da-Silva P. Long-term safety and efficacy of eslicarbazepine acetate as adjunctive therapy in the treatment of partialonset seizures in adults with epilepsy: results of a 1-year openlabel extension study. Epilepsy Res. 2013;103(2-3):262-9.

46. Villanueva V, Serratosa JM, Guillamon E, Garces M, Giraldez BG, Toledo $M$, et al. Long-term safety and efficacy of eslicarbazepine acetate in patients with focal seizures: results of the 1-year ESLIBASE retrospective study. Epilepsy Res. 2014;108(7):1243-52.

47. Villanueva V, Bermejo P, Montoya J, Toledo M, Gomez-Ibanez A, Garces M, et al. EARLY-ESLI study: long-term experience with eslicarbazepine acetate after first monotherapy failure. Acta Neurol Scand. 2016;. doi:10.1111/ane.12720 (Epub ahead of print).

48. Correia FD, Freitas J, Magalhaes R, Lopes J, Ramalheira J, Lopes-Lima J, et al. Two-year follow-up with eslicarbazepine acetate: a consecutive, retrospective, observational study. Epilepsy Res. 2014;108(8):1399-405.

49. Sperling MR, Harvey J, Grinnell T, Cheng H, Blum D. Efficacy and safety of conversion to monotherapy with eslicarbazepine acetate in adults with uncontrolled partial-onset seizures: a randomized historical-control phase III study based in North America. Epilepsia. 2015;56(4):546-55.

50. Jacobson MP, Pazdera L, Bhatia P, Grinnell T, Cheng H, Blum D. Efficacy and safety of conversion to monotherapy with 
eslicarbazepine acetate in adults with uncontrolled partial-onset seizures: a historical-control phase III study. BMC Neurol. 2015;15:46.

51. Sperling MR, French J, Jacobson MP, Pazdera L, Gough M, Cheng $\mathrm{H}$, et al. Conversion to eslicarbazepine acetate monotherapy: a pooled analysis of 2 phase III studies. Neurology. 2016;86(12):1095-102.

52. Efficacy of eslicarbazepine acetate versus controlled-release carbamazepine as monotherapy in patients with newly diagnosed partial-onset seizures (press release). American Academy of Neurology 68th Annual Meeting; 2016.

53. Almeida L, Minciu I, Nunes T, Butoianu N, Falcao A, Magureanu SA, et al. Pharmacokinetics, efficacy, and tolerability of eslicarbazepine acetate in children and adolescents with epilepsy. J Clin Pharmacol. 2008;48(8):966-77.

54. Ley M, Principe A, Jimenez-Conde J, Rocamora R. Assessing long-term effects of eslicarbazepine acetate on lipid metabolism profile, sodium values and liver function tests. Epilepsy Res. 2015;115:147-52.

55. Holtkamp M, McMurray R, Bagul M, Sousa R, Kockelmann E. Real-world data on eslicarbazepine acetate as add-on to antiepileptic monotherapy. Acta Neurol Scand. 2016;134(1):76-82.

56. Rocamora R. A review of the efficacy and safety of eslicarbazepine acetate in the management of partial-onset seizures. Ther Adv Neurol Disord. 2015;8(4):178-86.
57. Kim YS, Kim DW, Jung KH, Lee ST, Kang BS, Byun JI, et al. Frequency of and risk factors for oxcarbazepine-induced severe and symptomatic hyponatremia. Seizure. 2014;23(3):208-12.

58. Berghuis B, de Haan GJ, van den Broek MP, Sander JW, Lindhout D, Koeleman BP. Epidemiology, pathophysiology and putative genetic basis of carbamazepine- and oxcarbazepine-induced hyponatremia. Eur J Neurol. 2016;23(9):1393-9.

59. Singh RP, Asconape JJ. A review of eslicarbazepine acetate for the adjunctive treatment of partial-onset epilepsy. J Cent Nerv Syst Dis. 2011;3:179-87.

60. Sachdeo R, Karia R. Antiepileptic drugs and hyponatremia: supplement to Neurology Reviews. 2016. http://cdn4.imng.com/ fileadmin/qhi/nr/pdfs/NR_Hyponatremia_Suppl_1215_v6.pdf. Accessed 17 Jul 2017.

61. Gupta DK, Bhoi SK, Kalita J, Misra UK. Hyponatremia following esclicarbazepine therapy. Seizure. 2015;29:11-4.

62. Massot A, Vivanco R, Principe A, Roquer J, Rocamora R. Postauthorisation study of eslicarbazepine as treatment for drug-resistant epilepsy: preliminary results. Neurologia. 2014;29(2):94-101.

63. Peltola J, Holtkamp M, Rocamora R, Ryvlin P, Sieradzan K, Villanueva V. Practical guidance and considerations for transitioning patients from oxcarbazepine or carbamazepine to eslicarbazepine acetate: expert opinion. Epilepsy Behav. 2015;50:46-9. 